\title{
Psoriasis e implicaciones cardiovasculares
}

\author{
Psoriasis and cardiovascular implications
}

\author{
Itziar Borbolla Schega, * Daniel López Zertuche, ${ }^{\ddagger}$ Enrique Juan Díaz Greene, ${ }^{\S}$ Federico Leopoldo Rodríguez Weber ${ }^{\llbracket}$ \\ Citar como: Borbolla SI, López ZD, Díaz GEJ, Rodríguez WFL. Psoriasis e implicaciones cardiovasculares. \\ Acta Med Grupo Angeles. 2021; 19 (2): 253-257. https://dx.doi.org/10.35366/100450
}

\section{Resumen}

La psoriasis es un desorden inflamatorio sistémico e inmunomediado que afecta la piel y diversos órganos. Su etiología exacta se desconoce, aunque posee marcadores genéticos y epigenética predisponente, además del papel fundamental del sistema inmune en su progresión. Incide en distintos sistemas, tal que el tejido adiposo ha demostrado sufrir disfunción, llevando a la resistencia a la insulina y por ende al síndrome metabólico, propicia el desarrollo de aterosclerosis e incremento del riesgo cardiovascular. La inflamación es el principal factor fisiopatológico en ese daño a las arterias, por ello se ha estudiado la asociación de la IL-17 con la disfunción endotelial, alteraciones cardiovasculares y psoriasis, donde probablemente hay una correlación positiva según la evidencia disponible. En pacientes con ese trastorno de la piel, las enfermedades del corazón constituyen la primera o segunda causa de mortalidad, tanto que a nivel mundial es de $46 \%$. Existen perturbaciones en los varios tipos de colesterol, triglicéridos, lipoproteínas y en Apo A1, junto con la hinchazón autoinmune, promueve la obstrucción arterial y el aumento del peligro cardiovascular que es influido también por esas macromoléculas modificadas por oxidación y las lipoproteínas de baja densidad oxidada. Se ha observado que la aterosclerosis de la arteria coronaria es un componente de riesgo importante para la cardiopatía isquémica, y se ha encontrado que los afectados por psoriasis tienen una mayor prevalencia y gravedad en la enfermedad coronaria.

Palabras clave: Psoriasis, riesgo cardiovascular, síndrome metabólico, enfermedad arterial coronaria.

\section{INTRODUCCIÓN}

La psoriasis es un desorden inflamatorio sistémico e inmunomediado que incide en diversos órganos además de la piel. Dicha entidad afecta a un 3\% de la población mun-

\section{Abstract}

Psoriasis is an immune-mediated and systemic inflammatory disorder that affects the skin and various organs. Its exact etiology is unknown, although it has predisposing genetic and epigenetic markers, in addition to the fundamental role of the immune system in its progression. It has effect on different systems, therefore, the adipose tissue has been shown to suffer dysfunction leading to insulin resistance and therefore to the metabolic syndrome, development of atherosclerosis and increased cardiovascular risk. Inflammation is the main pathophysiological factor in atherosclerosis, therefore the association of IL-17 with endothelial dysfunction, cardiovascular diseases and psoriasis has been studied where there is probably a positive correlation, according to the available evidence. In patients with this illness, heart diseases are the first or second cause of mortality, so high that mortality worldwide is $46 \%$. There are alterations in the different types of cholesterol, triglycerides, lipoproteins and Apo $A 1$, which together with autoimmune inflammation develop atherosclerosis and increase cardiovascular risk, which is also promoted by oxidation-modified lipoproteins and oxidized lowdensity lipoproteins. Coronary artery atherosclerosis has been discovered to be an important risk component for ischemic heart disease, patients with psoriasis have been found to have a higher prevalence and severity in coronary heart disease.

Keywords: Psoriasis, cardiovascular risk, metabolic syndrome, coronary artery disease.
* Médico residente de medicina interna.

${ }^{\ddagger}$ Médico interno de pregrado.

$\S$ Profesor titular del curso de Medicina Interna.

"Profesor adjunto del curso de Medicina Interna.

Hospital Ángeles Pedregal, Facultad Mexicana de Medicina de la Universidad La Salle, Ciudad de México. dial aproximadamente. La etiología exacta se desconoce, aunque se han visto marcadores genéticos y la epigenética como predisponentes. El sistema inmune juega un rol crucial en la progresión de la enfermedad; las células inmunes innatas, humorales y mediadores inflamatorios representan 
un papel determinante para la progresión de la dolencia y sus diferentes etapas. ${ }^{1,2}$

Tal patología se ha asociado con artritis, uveítis y enfermedad inflamatoria intestinal. Sin embargo, se ha visto que este proceso inflamatorio puede perjudicar al tejido adiposo, especialmente a los depósitos que rodean al corazón y vasos sanguíneos. El resultado de la disfunción del tejido graso y resistencia a la insulina se suele manifestar en un síndrome metabólico, pues se ha visto que aflige de un 20 a $40 \%$ de los pacientes con este trastorno, señaladamente a aquellos que tienen un involucro articular. ${ }^{3,4}$

Los trastornos cardiovasculares abarcan entidades como la aterosclerosis, infarto agudo al miocardio, isquemia, arritmias y falla cardiaca. La asociación de la psoriasis con el riesgo de estas disfunciones se puede atribuir a las moléculas proinflamatorias que se liberan durante el proceso inflamatorio crónico, al igual que los tratamientos como los retinoides o ciclosporina, que favorecen la aparición de hiperlipidemia. ${ }^{1}$

\section{IL-17 A COMO FACTOR PREDISPONENTE}

La inflamación es el principal factor fisiopatológico en la aterosclerosis. El sistema inmune parece modular todas las fases de la aterotrombosis, desde la lesión temprana del endotelio y el inicio de la placa, hasta la aterosclerosis más avanzada que implica cambios en flujo autofágico, mitofágico y en la ruptura de la placa, esto dado por los mayores niveles de estrés oxidativo. ${ }^{5}$

La interleucina 17 (IL-17) es una citocina que forma parte de la inmunidad innata. La IL-17A y IL-17F están implicadas en la respuesta inmune de dicho padecimiento. Estas citoquinas inician un estado proinflamatorio en los tejidos y reclutamiento de los neutrófilos. Existe evidencia que indica que IL-17A puede representar uno de los principales vínculos entre las manifestaciones de enfermedades cardiovasculares y la psoriasis. ${ }^{6}$

Las células T17 activadas en la piel producen diferentes citocinas, incluida IL-17 (IL-17A/IL-17F), TNF $\alpha$, IL-26 e IL29. IL-17, la principal citocina efectora en la psoriasis, actúa sola o sinérgicamente con TNF $\alpha$ para inducir la expresión y liberación de mediadores de hinchazón incluidas hBD2, LCN2, proteínas S100 y LL37/catelicidina. Los efectos de la IL-17 sobre los queratinocitos epidérmicos producen una hinchazón en las placas psoriásicas maduras. La IL-22, IL-19 e IL-36 en réplica a IL-17 contribuyen al desarrollo de hiperplasia epidérmica dándole al tejido cutáneo un aspecto engrosado y escamoso con núcleos retenidos, en el estudio histopatológico. La piel por sí sola también sintetiza IL-17C en respuesta a IL-17A/F y tal citocina amplifica muchos genes asociados a esta enfermedad crónica que también pueden estimular a las células T17 y aumentar la producción de IL-17A/F.5,6
La fisiopatología de la psoriasis implica mediadores inflamatorios tales como el factor de necrosis TNF $\alpha$, IL-1B, IL-6, IL-12, IL-17A, IL-17F e IL-23, liberados principalmente por ayudante activado tipo 1 y ayudante tipo $17 \mathrm{~T}$ (Th17) y células dendríticas. La IL-17A está implicada en vías fisiopatológicas comunes entre esa afección cutánea y los padecimientos cardiovasculares y tiene acción proaterogénica. ${ }^{7}$

La sobreexpresión de IL-17A trae como consecuencia altos niveles de células inflamatorias circulantes, esto promueve la disfunción e inflamación vascular, estrés oxidativo y la hipertrofia del ventrículo izquierdo.

La mencionada citocina está asociada con aterosclerosis y disfunción endotelial ya que la IL-17 induce la expresión de IL-6 en fibroblastos e IL-6 induce la diferenciación de células Th17 responsables de la liberación de IL-17A. Por lo tanto, resulta en una retroalimentación positiva activada por IL-17 y seguida por la expresión de IL-6. La IL-6 se encuentra aumentada en psoriasis, y se ha verificado que tiene un efecto inotrópico negativo y está asociado con aumento de la rigidez arterial e isquemia miocárdica. ${ }^{`}$

\section{PSORIASIS Y ENFERMEDADES CARDIOVASCULARES}

La enfermedad cardiovascular y la psoriasis están ampliamente relacionados; según los resultados informados en estudios epidemiológicos, los padecimientos cardiovasculares constituyen la primera o segunda causa de mortalidad en pacientes con la afección inflamatoria. La duración también se ha asociado con tal riesgo. En términos generales, la mortalidad a nivel mundial es de $46 \%$, cuenta con una tasa del 1.44 (IC del 95\%: 1.43-1.45) por 1,000 años-persona. De acuerdo con estos hallazgos, personas con el trastorno cutáneo tienen un mayor peligro de eventos cardiovasculares, en particular en aquellos con un trastorno severo, el RR fue de 3.04 para infarto y 1.59 para accidente cerebrovascular. La psoriasis severa confiere un riesgo absoluto adicional de $6.2 \%$ de una tasa de enfermedades cardiovasculares a 10 años, comparado con la población general. ${ }^{8,9}$

El patrón lipídico consiste en mayores concentraciones de colesterol en las lipoproteínas de muy baja densidad (LDL) y fracciones de lipoproteínas de alta densidad (HDL), al inicio del padecimiento, se han confirmado valores más altos de Apo A1; esto juega un papel perjudicial de HDL-C y Apo A1 causado por estrés oxidativo. Los niveles de lipoproteína se encuentran elevados en esta enfermedad y se correlacionan con marcadores de estrés oxidativo. Existen niveles significativamente más bajos de HDL-c y más altas marcas de colesterol total, colesterol LDL y triglicéridos (TG), estas anormalidades lipídicas actúan en conjunto con la turgencia autoinmune y promueven la formación de aterosclerosis y el aumento del riesgo cardiovascular, dados por la vía que comparten con la modulación de monocitos 
y neutrófilos, disfunción del endotelio y señalización del eje IL-23/Th17 (Figura 1). ${ }^{8}$

Se cree que hay vías de inflamación endógenas compartidas entre ambas entidades, dentro de los mecanismos inmunológicos, los más relevantes son IL-12/Th1 e IL-23/ Th17, que conducen al crecimiento de la placa, promovido por TNF $\alpha$ e IFN-c de Th1 diferenciado. , $^{8,10}$

La aterosclerosis es el principal cambio patológico que precede al infarto agudo al miocardio y al accidente cardiovascular. Se han realizado estudios en donde se demuestra que el grado de hinchazón vascular está estrechamente relacionado con la gravedad de la psoriasis. Se ha encontrado que una reducción en la severidad del padecimiento de psoriasis está asociada con una mejora en aterosclerosis coronaria. ${ }^{11}$

La aterosclerosis de dicha arteria es un factor de contingencia importante para la cardiopatía isquémica, se ha descubierto que los pacientes con psoriasis revelan una mayor prevalencia y gravedad en la enfermedad coronaria, calcificación arterial y aterosclerosis. El desarrollo de los daños en la coronaria en enfermos con psoriasis puede estar parcialmente relacionado con el deterioro de la capacidad de salida de colesterol de los macrófagos. ${ }^{11}$

La aterosclerosis de la carótida se reconoce como un ingrediente de peligro hacia el desarrollo de un evento cerebrovascular. Se ha demostrado que los pacientes con psoriasis tienen un mayor grosor de la íntima media carotídea en comparación con aquellos sin esta patología. ${ }^{11}$

El riesgo cardiovascular en afectados con psoriasis está dado por el efecto inflamatorio de las lipoproteínas mo- dificadas por oxidación (OML) y las lipoproteínas de baja densidad oxidada (oxLDL), ya que éstas juegan un papel importante en la activación de las células endoteliales, la formación de células espumosas de los macrófagos y la migración y proliferación de células musculares lisas. La lipoproteína de alta densidad, en los pacientes sin la afección cutánea, juega un rol protector, sin embargo, en los que padecen psoriasis se puede convertir en oxHDL (HDL oxidado) y tal mudanza lo convierte a un perfil aterogénico, al igual que una disminución de flujo de colesterol, y esto favorecer a la formación de placas aterogénicas y aparición de síndromes coronarios agudos. ${ }^{12}$

\section{SIINDROME METABÓLICO, ATEROSCLEROSIS Y PSORIASIS}

El síndrome puede ocurrir transitoriamente en personas sin obesidad durante la enfermedad, ya que, al aumentar la secreción de TNF $\alpha$, IL-6 e IL-1 $\beta$ se induce un estado temporal de resistencia a la insulina, si la inflamación persiste esto puede volverse crónico, dada la infiltración de macrófagos y células T en el tejido adiposo. Por otro lado, la obesidad visceral y la resistencia a la insulina se caracterizan por la producción persistente de adipocitocinas anormales como TNF $\alpha$, IL-6, IL-1 $\beta$, leptina y adiponectina, que contribuyen al desarrollo de un estado proinflamatorio conduciendo además a una turgencia vascular crónica subclínica que modula y produce procesos ateroscleróticos. ${ }^{13}$

La aterosclerosis se define como un padecimiento inflamatorio crónico mediado por el sistema inmune que surge

Figura 1: Vías de inflamación compartidas entre ambas entidades, dentro de los mecanismos inmunológicos, los más relevantes son IL-12/Th1 e IL-23/Th17, que conducen al crecimiento de la placa y síndrome metabólico.

Esquema sobre las vías de inflamación sistémica en la psoriasis.

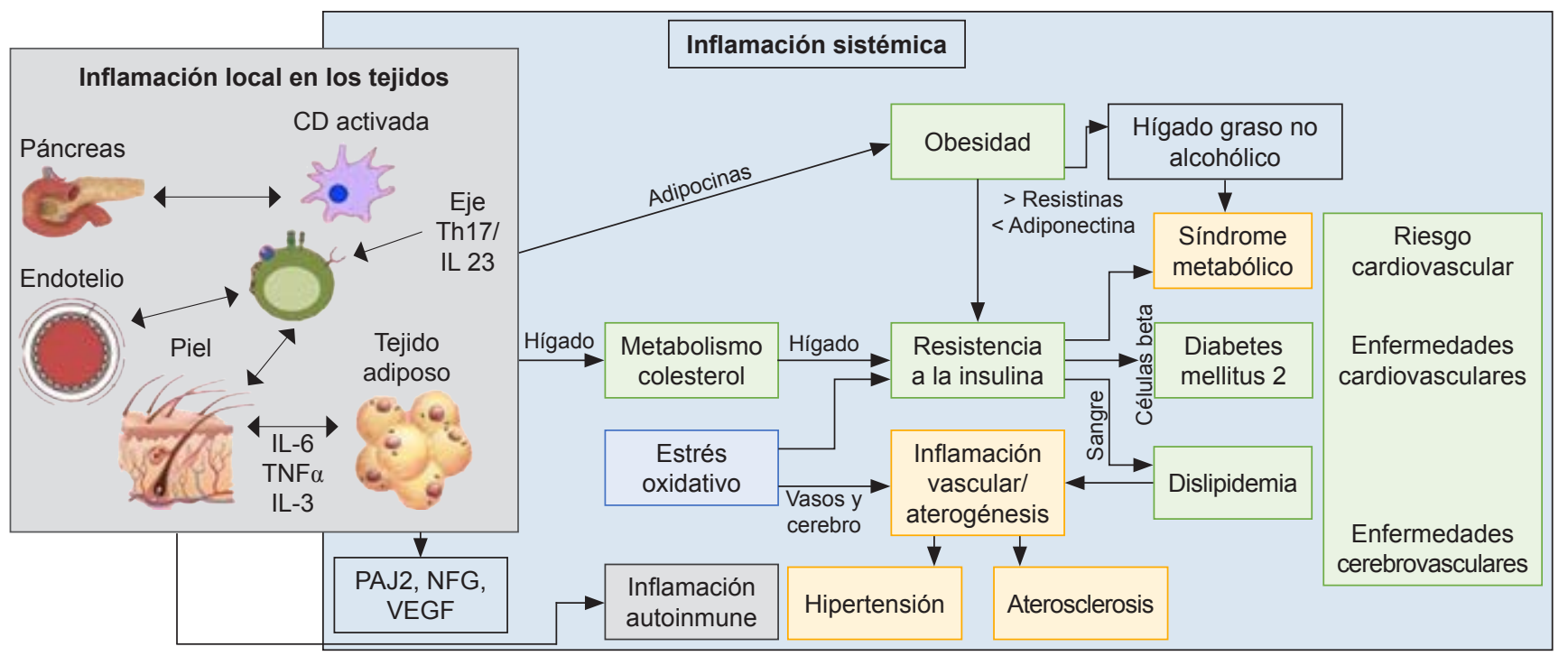


de una serie de eventos complejos que se desencadenan por disfunción endotelial, depósitos de lípidos en la pared arterial y la infiltración de macrófagos derivados de monocitos. Las citocinas, en especial IL-1 $\beta, \mathrm{TNF} \alpha$, IL-6 e IL-17 se consideran patógenamente relevantes y susceptibles a la intervención terapéutica. ${ }^{13}$

\section{FACTOR DE RIESGO CARDIOVASCULAR Y PSORIASIS}

En la actualidad, se ha agregado a la escala de riesgo de Framingham la psoriasis. Esto reclasificó a la mayoría de los pacientes en una categoría de riesgo cardiovascular más elevado. El peligro asociado a psoriasis severa es equiparable con enfermedades crónicas como la diabetes. ${ }^{9}$

Los aquejados con esa dolencia crónica alcanzan una puntuación de Framingham significativamente mayor; un estudio de pacientes italianos con psoriasis en controles mostraron que tenían un porcentaje absoluto de $5.3 \%$ a los cinco años, y $11.2 \%$ a los 10 años, de desarrollar un evento mayor (infarto de miocardio o accidente cerebrovascular). Rosa y colaboradores calcularon el riesgo cardiovascular proyectado a los 10 años, según los criterios de Framingham en 89 pacientes con psoriasis, el $68.4 \%$ de quienes tenían bajo riesgo, $18.4 \%$ en el nivel moderado y $13.3 \%$ en el alto. ${ }^{14}$

Por otro lado, está el estudio "Riesgo cardiovascular en la psoriasis: un análisis basado en la población con evaluación de la escala de Framingham", en el cual se analizaron los datos obtenidos de 1,421 pacientes distribuidos en dos grupos; el primero de 224 personas que consistió en observación por un periodo aproximado de 10 años, y el segundo con 1,197 integrantes en el que se llevó a cabo la evaluación por dicha escala de riesgo de Framingham. La conclusión más relevante de este estudio comparativo es que el score utilizado no demuestra ventaja alguna sobre solamente observar a los enfermos durante su evolución, por lo cual no se incluye la recomendación del mismo de manera rutinaria. ${ }^{15}$

La enfermedad coronaria es la condición cardiovascular más prevalente en psoriasis, que varía de $4.6 \%$ a $7.8 \%$; la predominancia del trastorno cerebrovascular de $3.1 \%$ a $6.5 \%$ y la vascular periférica, que varía del $2.7 \%$ a $4.9 \%$. La presencia de sobrepeso osciló entre $22 \%$ a $37 \%$, si se toma en cuenta la severidad de la afección cutánea la prevalencia de sobrepeso varía, en la psoriasis leve es de $34 \%$ a $35 \%$, de moderada a severa la misma varió de $35 \%$ a $40 \%$. En la categoría severa, la prevalencia de diabetes mellitus fue de $41.9 \%$. La hipertensión arterial sistémica se encontró estrechamente relacionada, teniendo una relevancia de $31 \%$ en la psoriasis leve y $40.3 \%$ en la severa. La dislipidemia varió ampliamente entre $6.4 \%$ y $50.9 \%$ en psoriasis (gravedad no especificada), 23.9\% en la leve y $29.9 \%$ cuando era severa. ${ }^{16}$

\section{ESTRATEGIAS PARA REDUCIR EL RIESGO CARDIOVASCULAR}

La interleucina (IL) 17A se ha identificado como la citocina principal en la inflamación inducida por la psoriasis y de la enfermedad vascular. La citocina también ha sido implicada en la patobiología del padecimiento vascular. El impacto de la inhibición de IL-17 A, con el uso de secukinumab mejoró la reserva de flujo coronario (CFR), la velocidad de la onda de pulso (PWV) y las medidas de estrés oxidativo. Los niveles elevados de IL-17 A/F se han encontrado en los pacientes que presentan placas ateroescleróticas avanzadas, en enfermos con infarto agudo al miocardio y con angina inestable. Los antagonistas de IL-17 podrían desempeñar un papel en la reducción de la morbimortalidad de los enfermos con el trastorno de la piel. ${ }^{6,17}$

Actualmente, la terapia con inhibidores de IL-12 e IL-23 como el ustekinumab, se encuentra siendo evaluada ya que en un estudio mostró que, aparte de haber disminuido el índice de la severidad del área de psoriasis (PASI), disminuyó la hinchazón de otros tejidos, entre éstos, el de las estructuras vasculares, dando pie a que en futuras investigaciones se evalúe su capacidad cardioprotectora. ${ }^{18}$

También, dentro de estos nuevos tratamientos para reducir el riesgo cardiovascular en pacientes con psoriasis, se está prestando atención al canakinumab (inhibidor de IL-1B) que en ensayos clínicos ha confirmado tener un efecto cardioprotector, pudiendo prevenir eventos vasculares recurrentes en enfermos con infarto agudo al miocardio previo, pues tienen una respuesta proinflamatoria persistente. ${ }^{19,20}$

\section{CONCLUSIÓN}

A través de esta revisión se puede determinar que existe una fuerte correlación entre la psoriasis y el riesgo cardiovascular en estos pacientes. Se ha determinado que el principal factor para el desarrollo de la aterosclerosis es el inflamatorio, el cual se ha ligado principalmente a las interleucinas antes expuestas, siendo la más importante la IL-17 junto con la participación de vías endógenas de inflamación donde interactúan IL-12/Th1 e IL-23/Th17 que conducen a alteraciones en lipoproteínas, el estrés oxidativo y la disfunción endotelial. Hoy en día, el poder estratificar el peligro cardiovascular en esta población continúa representando un reto, que con el advenimiento de futuros estudios facilitaran su determinación, sin embargo, es clara la influencia de esa enfermedad en el desarrollo de otras entidades patológicas como son las cardiovasculares, cerebrovasculares y vascular periférica, al igual que diabetes mellitus e hipertensión arterial sistémica. 
Finalmente, buscando mejorar e incluso evitar estas comorbilidades se ha determinado que las citocinas como IL-1 $\beta$, TNF, IL-6 e IL-17 son susceptibles a la intervención terapéutica; la inhibición de IL-17A, IL-12 e IL-23 ha revelado un efecto prometedor al disminuir la hinchazón vascular, entre otros logros, y se está evaluando la posibilidad del uso de nuevos fármacos, esto último se sabrá con el resultado de los futuros ensayos clínicos en esta materia.

\section{REFERENCIAS}

1. Choudhary S, Patel R, Pradhan D, Deval R, Singh H, Thomas $\mathrm{G}$ et al. Psoriasis and cardiovascular disorders: association or epiphenomenon? Meta-analysis of observational studies. 3 Biotech. 2020; 10 (3): 104.

2. Espinoza HCJ, Kresch TN, Vega MME, Lacy NRM, Lacy NMC, Springall VR et al. Psoriasis y riesgo cardiovascular. Uso de la escala de riesgo Framingham y nuevos biomarcadores para la predicción de mortalidad cardiovascular en pacientes con psoriasis. Dermatología Cosmética, Médica y Quirúrgica. 2012; 10 (4): 301.

3. Packer M. Epicardial adipose tissue inflammation can cause the distinctive pattern of cardiovascular disorders seen in psoriasis. Am J Med. 2020; 133 (3): 267-272.

4. Curco N, Barriendos N, Barahona MJ, Arteaga C, García M, Yordanov $\mathrm{S}$ et al. Factors influencing cardiometabolic risk profile in patients with psoriasis. Australas J Dermatol. 2018; 59 (2): e93-e98.

5. Verma S, Bhatt DL. Does interleukin-17A blockade have a potential clinical role to reduce cardiovascular risk in psoriasis? Can J Cardiol. 2020; 36 (1): 24-26.

6. Von Stebut E, Boehncke WH, Ghoreschi K, Gori T, Kaya Z, Thaci D et al. IL-17A in psoriasis and beyond: cardiovascular and metabolic implications. Front Immunol. 2019; 10: 3096.

7. Makavos G, Ikonomidis I, Andreadou I, Varoudi M, Kapniari I, Loukeri $\mathrm{E}$ et al. Effects of interleukin $17 \mathrm{~A}$ inhibition on myocardial deformation and vascular function in psoriasis. Can J Cardiol. 2020; 36 (1): 100-111.

8. Egeberg A, Gisondi P, Carrascosa J, Warren R, Mrowietz U. The role of the IL-23/Th17 pathway in cardio-metabolic comorbidity associated with psoriasis. J Eur Acad Dermatol Venereol. 2020; 34 (8): 1695-1706.
9. Takeshita J, Grewal S, Langan SM, Mehta NN, Ogdie A, Van Voorhees AS et al. Psoriasis and comorbid diseases: epidemiology. J Am Acad Dermatol. 2017; 76 (3): 377-390.

10. Asha K, Singal A, Sharma SB, Arora VK, Aggarwal A. Dyslipidaemia \& oxidative stress in patients of psoriasis: Emerging cardiovascular risk factors. Indian Journal of Medical Research. 2017; 146 (6): 708-713.

11. Hu SC-S, Lan C-CE. Psoriasis and cardiovascular comorbidities: focusing on severe vascular events, cardiovascular risk factors and implications for treatment. Int J Mol Sci. 2017; 18 (10): 2211.

12. Siddiqi HK, Ridker PM. Psoriasis and atherosclerosis: where inflammation and lipid biology meet. Circulation Research. 2018; 123: 1183-1184.

13. Puig L. Cardiometabolic comorbidities in psoriasis and psoriatic arthritis. Int J of Mol Sci. 2018; 19 (1): 58.

14. Fernández-Torres R, Pita-Fernández S, Fonseca E. Psoriasis and cardiovascular risk. Assessment by different cardiovascular risk scores. J Eur Acad Dermatol Venereol. 2013; 27 (12): 1566-1570.

15. Myasoedova E, Manu AVB, Matteson EL, Maradit KH, McEvoy MT, Crowson CS. Cardiovascular risk in psoriasis: a population-based analysis with assessment of the Framingham risk score. Scientifica (Cairo). 2013; 2013: 371569.

16. Daudén E, Castañeda S, Suárez C, García-Campayo J, Blasco A, Aguilar $\mathrm{M}$ et al. Clinical practice guideline for an integrated approach to comorbidity in patients with psoriasis. J Eur Acad Dermatol Venereol. 2013; 27 (11): 1387-1404.

17. Wu D, Hou SY, Zhao S, Hou LX, Jiao T, Xu NN et al. Efficacy and safety of interleukin-17 antagonists in patients with plaque psoriasis: a meta-analysis from phase 3 randomized controlled trials. J Eur Acad Dermatol Venereol. 2017; 31 (6): 992-1003.

18. Kim BS, Lee WK, Pak K, Han J, Kim GW, Kim HS et al. Ustekinumab treatment is associated with decreased systemic and vascular inflammation in patients with moderate to severe psoriasis: Feasibility study using $18 \mathrm{~F}$-fluorodeoxyglucose positron emission tomographycomputed tomography. J Am Acad Dermatol. 2019; 80 (5): 13221331. doi: 10.1016/j.jaad.2018.03.011.

19. Ridker PM, Everett BM, Thuren T, MacFadyen JG, Chang WH, Ballantyne $C$ et al. Antiinflammatory therapy with canakinumab for atherosclerotic disease. N Engl J Med. 2017; 377 (12): 1119-1131.

20. Skendros P, Papagoras C, Lefaki I, Giatromanolaki A, Kotsianidis I, Speletas $\mathrm{M}$ et al. Successful response in a case of severe pustular psoriasis after interleukin-1 $\beta$ inhibition. Br J Dermatol. 2017; 176 (1): 212-215.

Conflicto de intereses: Los autores manifiestan no contar con conflicto de intereses en la realización de este trabajo. 